\title{
Mechanisms behind the Faceting of Catanionic Vesicles by Polycations: Chain Crystallization and Segregation
}

\author{
Filipe E. Antunes, ${ }^{\dagger}$ Rodrigo O. Brito ${ }^{\ddagger}$ Eduardo F. Marques,,$\stackrel{\star}{\ddagger}$ Björn Lindman, ${ }^{\dagger, \S}$ and \\ Maria Miguel ${ }^{\dagger}$ \\ Chemistry Department, University of Coimbra, 3004-535 Coimbra, Portugal, Centro de Investigação em \\ Química, Department of Chemistry, University of Porto, Rua do Campo Alegre, $n^{\circ}$ 687, P-4169-007 Porto, \\ Portugal, and Physical Chemistry 1, Center for Chemistry and Chemical Engineering, Lund University, \\ P.O. Box 124, SE-221 00 Lund, Sweden
}

Received: June 26, 2006; In Final Form: October 31, 2006

\begin{abstract}
Vesicles composed of an anionic and a cationic surfactant, with a net negative charge, associate strongly with a hydrophobically modified polycation (LM200) and with an unmodified polycation with higher charge density (JR400), forming viscoelastic gel-like structures. Calorimetric results show that in these gels, LM200 induces a rise of the chain melting temperature $\left(T_{\mathrm{m}}\right)$ of the vesicles, whereas JR400 has the opposite effect. For both polymer-vesicle systems, the shear viscosity exhibits an inflection point at $T_{\mathrm{m}}$, and for the LM200 system the measured relaxation times are significantly higher below $T_{\mathrm{m}}$. The neat vesicles and the polycationbound vesicles have a polygonal-like faceted shape when the surfactant chains in the bilayer are crystallized, as probed by cryo-transmission electron microscopy. Above $T_{\mathrm{m}}$, the neat and the LM200-bound vesicles regain a spheroidal shape, whereas those in the JR400 system remain with a deformed faceted shape even above $T_{\mathrm{m}}$. These shape changes are interpreted in terms of different mechanisms for the polymer-vesicle interaction, which seem to be highly dependent on polymer architecture, namely charge density and hydrophobic modification. A crystallization-segregation mechanism is proposed for the LM200-vesicle system, while, for the JR400-vesicle one, charge polarization-lateral segregation effects induced by the polycation in the catanionic bilayer are envisaged.
\end{abstract}

\section{Introduction}

Polymer-vesicle systems are colloidal systems of great interest in connection, for instance, with modeling studies for biomembranes, ${ }^{1-6}$ the design of efficient drug ${ }^{7,8}$ and gene delivery ${ }^{9,10}$ systems, and the rheological control of materials. ${ }^{11,12}$ When a polyelectrolyte is mixed with oppositely charged vesicles, the outcome of the association depends on several parameters, such as the vesicle size and concentration, the flexibility of the polyelectrolyte backbone, its charge density, and the ionic strength of the medium. The polyelectrolyte adsorbs strongly onto the vesicles, resulting in polyion-coated vesicles or in charge inversion, if more polyions collapse in the charged surface than are necessary to neutralize it. ${ }^{13}$ The adsorption may induce shifts in the gel-to-liquid crystal transition temperature of the vesicle, ${ }^{14,15}$ shape changes, ${ }^{16}$ or breakage of the vesicles. ${ }^{17}$ Addition of a polyelectrolyte to a charged bilayer has also been experimentally ${ }^{18}$ and theoretically ${ }^{19}$ shown to promote lateral segregation of charges. Another feature of the polymer-vesicle association, at sufficiently high concentration of both polyion and aggregates, is gelation, i.e., formation of a three-dimensional network of aggregates bridged by the polymer chains. ${ }^{12,20-23}$

The shape changes induced by the polymer can be temperature-dependent and directly connected with the gel-to-liquid crystal phase transition of the vesicles, often also simply

\footnotetext{
* Corresponding author. E-mail: efmarque@fc.up.pt

University of Coimbra.

$\doteqdot$ University of Porto.

$\S$ Lund University.
}

designated as chain melting transition. It is known that planar hydrated bilayers and discrete bilayer aggregates with non-zero curvature (such as vesicles), formed by double-chained amphiphiles, exhibit this type of first-order phase transition. Above the transition temperature, $T_{\mathrm{m}}$, the amphiphile has fluid disordered alkyl chains and vesicles typically exhibit spheroidal shape. Below this temperature, upon crystallization of the chains and the curvature constraints imposed by it, nonspheroidal aggregates can be formed ${ }^{24-28}$ (e.g., disks, planar bilayer fragments, lens-shaped vesicles, regular polygon-shaped vesicles, and irregularly faceted vesicles), while reheating restores the spheroidal shape. In two-component catanionic vesicles, segregation has been shown to occur in the frozen state and be responsible for the observed polygonal-shaped vesicles. ${ }^{29}$

In previous work we have shown that two different cationic polyelectrolytes have significant and interesting effects on the structure of catanionic vesicles, ${ }^{30}$ with a net negative charge. ${ }^{12,21}$ These types of vesicles have been shown to have long-term equilibrium stability and be suitable models for biomembranes and drug delivery systems. ${ }^{31,32}$ The polycations used have different charge densities, and one is hydrophobically modified. The polymers associate with the vesicles forming threedimensional networks ("gels") and leading to the appearance of faceted vesicles, by a mechanism that is still not clarified and deserves closer investigation. For the interaction at stake, three main possibilities arise, depending on the polycation properties (essentially, charge density and presence of hydrophobic groups): (i) The polycation induces surfactant crystallization, shifting the $T_{\mathrm{m}}$ to higher value. (ii) The polycation induces lateral segregation within the vesicle, without loss of 

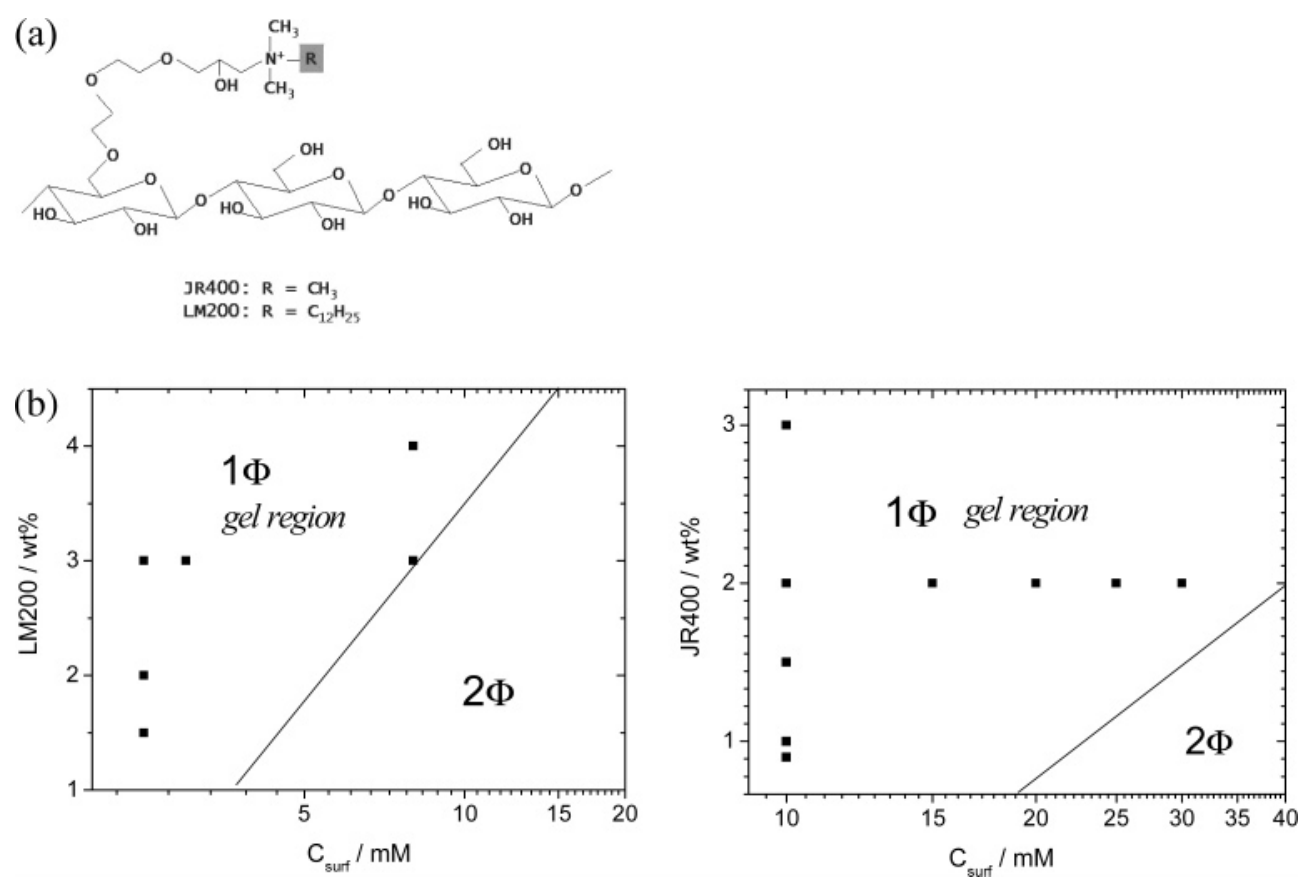

Figure 1. Polymer-vesicle system investigated in this work: (a) structure of the two polymers; (b) phase maps of the LM200-vesicle system and JR400-vesicle system (adapted from ref 21). The vesicles consist of a SDS-DDAB mixture at $X_{\mathrm{SDS}}=0.71$. The samples in the gel region studied in this work are indicated as squares. The straight line is the phase boundary between the single-phase gel region and the two-phase precipitate region. To be noted is that the term gel here designates the polymer-vesicle rheological gel. It should be distinguished from the gel phase of the vesicle bilayer, i.e., bilayer aqueous phase where the surfactant alkyl chains are in a crystalline state.

integrity, forming anionic rich domains coexisting with cationic rich domains. (iii) The polycation leads to the disintegration of the catanionic vesicles, promoting the formation of polymeranionic surfactant micelles and cationic surfactant-enriched aggregates. The aim of this paper is to show experimental evidence to discern between these different mechanisms and draw some general conclusions on the relation between polymer architecture and type of structural effect.

\section{Experimental Section}

2.1. Materials. High-purity surfactants sodium dodecylsulfate (SDS) and didodecyldimethylammonium bromide (DDAB) were supplied by Aldrich; dodecyltrimethylammonium bromide (DTAB), used as model system for comparison with LM200, was supplied by Sigma. The surfactants were used as received, after expected cmc values were obtained by surface tension (du Noüy ring method). The two different cationic polyelectrolytes, JR400 and LM200 (Figure 1a), are hydroxyethyl cellulose derivatives manufactured by Union Carbide Chemicals and Plastics Co., Inc.; 1 wt \% aqueous solutions of JR400 and of LM200 have charge concentration of ca. 10 and $2 \mathrm{mM}$, respectively, as determined by titration, ${ }^{33-35}$ implying a chargeto-charge contour length of 2 and $10 \mathrm{~nm}$, respectively. LM200 also contains grafted dodecyl chains, and the degree of hydrophobic modification is about 5.4 hydrophobic chains/100 sugar residues. ${ }^{34,35}$ Hydrophobically modified hydroxyethyl cellulose, HM-HEC, also used in some samples for comparison with the LM200 system, is supplied by AkzoNobel Surface Chemistry. Each polymer chain has 12 hydrophobic groups, corresponding to a degree of hydrophobic modification of $0.76 \%$, as given by the manufacturer.

2.2. Methods. Sample Preparation. A $55 \mathrm{mM}$ surfactant stock solution, with $C_{\text {surf }}=C_{\mathrm{SDS}}+C_{\mathrm{DDAB}}$, with a molar fraction of SDS, $X_{\mathrm{SDS}}=C_{\mathrm{SDS}} / C_{\text {surf }}=0.71$, was prepared by thoroughly mixing a SDS micellar solution and a DDAB homogenized dispersion. The resulting bluish solution contains a population of unilamellar vesicles bearing anionic net charge. ${ }^{30}$ This solution was sonicated for $5 \mathrm{~min}$ at $25{ }^{\circ} \mathrm{C}$, with a $200 \mathrm{~W}$ tipsonicator at $50 \%$ of the duty cycle. The surfactant-polymer samples were prepared by mixing the solid polymer and vesicle solution in appropriate amounts, to obtain samples in the gel region of the phase maps, as can be seen in Figure 1b, redrawn from ref 21. Samples along lines with constant surfactant content and varying polymer content, or constant polymer content and varying surfactant content, were prepared. The samples were vortexed, heated to ca. $50{ }^{\circ} \mathrm{C}$ for approximately $30 \mathrm{~min}$, and revortexed at room temperature. Upon heating, the viscosity of the samples decreases, which facilitates mixing. In samples containing LM200, it has been found that, without heating, a mixture of cloudy domains and clear gel areas appears. ${ }^{21}$ After five repeated cycles (mixing-heating-mixing), depending on sample viscosity, the samples were centrifuged at $25{ }^{\circ} \mathrm{C}$ for approximately $6 \mathrm{~h}$ to remove air bubbles. Samples are labeled as Sx, LySx, or JySx with S, L, and J denoting respectively surfactant, LM200, and JR400 and x and y denoting respectively surfactant concentration in $\mathrm{mM}$ and polymer wt \%. Samples with HMHEC and DTAB were labeled as HySx and DySx, respectively, following the same nomenclature.

Differential Scanning Calorimetry. DSC scans were made using a Setaram microDSCIII high-sensitivity calorimeter. Two steel batch cells were used, one containing ca. $0.8 \mathrm{~mL}$ of sample and the other, filled with the same amount of water, used as reference. The chain melting transition temperatures listed in Table 1 correspond to the maximum of the main peak, and the enthalpies mentioned in the text were calculated from the integration of the area under the peak, by means of the software provided by the manufacturer. All samples were scanned at $1 \mathrm{~K} \mathrm{~min}^{-1}$, and the reported results refer to upscans (endothermic peaks).

Cryo-TEM. Cryogenic transmission electron microscopy (cryo-TEM) studies were performed at 9,25 , and $30{ }^{\circ} \mathrm{C}$, following well-established procedures. ${ }^{36}$ Before film preparation, 
TABLE 1: Chain Melting Temperatures of Neat SDS/DDAB Vesicles and Those in the Presence of Polymers LM200, JR400 and HMHEC Surfactant DTAB ${ }^{a}$

\begin{tabular}{|c|c|c|c|c|c|c|c|c|c|c|c|c|c|}
\hline \multicolumn{2}{|c|}{ SDS/DDAB vesicles } & \multicolumn{3}{|c|}{ LM200-SDS/DDAB vesicles } & \multicolumn{3}{|c|}{ JR400-SDS/DDAB vesicles } & \multicolumn{3}{|c|}{ HMHEC-SDS/DDAB vesicles } & \multicolumn{3}{|c|}{ DTAB-SDS/DDAB vesicles } \\
\hline sample & $T_{\mathrm{m}} /{ }^{\circ} \mathrm{C}$ & sample & $T_{\mathrm{m}} /{ }^{\circ} \mathrm{C}$ & $\Delta T_{\mathrm{m}} /{ }^{\circ} \mathrm{C}$ & sample & $T_{\mathrm{m}} /{ }^{\circ} \mathrm{C}$ & $\Delta T_{\mathrm{m}} /{ }^{\circ} \mathrm{C}$ & sample & $T_{\mathrm{m}} /{ }^{\circ} \mathrm{C}$ & $\Delta T_{\mathrm{m}} /{ }^{\circ} \mathrm{C}$ & sample & $T_{\mathrm{m}} /{ }^{\circ} \mathrm{C}$ & $\Delta T_{\mathrm{m}} /{ }^{\circ} \mathrm{C}$ \\
\hline S2.5 & 21.0 & L2S 2.5 & 23.9 & +2.9 & $\mathrm{~J} 1 \mathrm{~S} 10$ & 14.7 & -3.8 & H1S8 & 18.2 & -1.8 & $\mathrm{D} 2 \mathrm{~S} 2.5$ & 24.8 & +6.3 \\
\hline S5 & 21.3 & L3S2.5 & 23.9 & +2.9 & $\mathrm{~J} 1.5 \mathrm{~S} 10$ & 14.5 & -4.0 & H3S8 & 17.8 & -2.2 & D3S2. 5 & 24.4 & +5.9 \\
\hline S8 & 20.0 & L3S3 & 23.7 & +2.6 & $\mathrm{~J} 2 \mathrm{~S} 10$ & 14.6 & -3.9 & & & & D3S3 & 24.6 & +6.1 \\
\hline $\mathrm{S} 10$ & 18.5 & L2.5S8 & 23.7 & +3.7 & $\mathrm{~J} 3 \mathrm{~S} 10$ & 14.2 & -4.3 & & & & D2.5S8 & 25.4 & +6.9 \\
\hline S20 & 16.1 & L3S8 & $22.6(38.1)$ & +2.6 & $\mathrm{~J} 2 \mathrm{~S} 15$ & 14.4 & -4.1 & & & & D3S8 & 25.1 & +6.6 \\
\hline S30 & 14.4 & & & & $\mathrm{~J} 2 \mathrm{~S} 20$ & 13.8 & -2.1 & & & & D3S10 & 25.3 & +6.8 \\
\hline S40 & 13.6 & & & & $\mathrm{~J} 2 \mathrm{~S} 25$ & 13.4 & -1.6 & & & & & & \\
\hline & & & & & $\mathrm{J} 2 \mathrm{~S} 30$ & 13.2 & -1.2 & & & & & & \\
\hline
\end{tabular}

${ }^{a}$ For sample notation, see caption of Figure 2. $\Delta T_{\mathrm{m}}=T_{\mathrm{m}}$ (polymer-vesicle or DTAB-vesicle system) $-T_{\mathrm{m}}($ neat vesicle). The uncertainty in $T$ values is $\pm 0.1^{\circ} \mathrm{C}$

the samples were let to rest at least $24 \mathrm{~h}$ at around the measurement temperature. For each temperature, the samples were placed in a vitrification chamber and a small drop was put on a copper grid supported carbon-coated holey film. The samples were then plunged into liquid ethane (at its melting temperature). The vitrified film was then transferred under liquid nitrogen to a Philips CM 120 Bio-Twin electron microscope and the imaging carried out.

Rheology. Rheological measurements were performed by using a Reologica Stress Tech rheometer equipped with an automatic gap setting. Cone geometry was employed, and to minimize evaporation, a solvent trap system was used. The temperature was controlled by a water bath. Prior to any oscillatory test, the linear viscoelastic region for each sample was determined by stress sweep tests. Measurements within the linear region ensure that the measured rheological properties are independent of the applied stress and that (near) equilibrium properties are probed. Below $T_{\mathrm{m}}$, the studied systems relax at very low frequencies. Relaxation times were found by applying power law equations to the frequency dependence of storage and loss modulus, $G^{\prime}=K f^{n}$ and $G^{\prime \prime}=K f^{n}$, within the range of $0.01-1 \mathrm{~Hz}$. The frequency at which storage and loss modulus intersect is easily given by equalizing the two equations. Relaxation times were then calculated from the inverse of the angular frequency. ${ }^{37} \mathrm{In}$ addition to the oscillatory measurements, shear viscosity as a function of temperature $\left(5-50{ }^{\circ} \mathrm{C}\right)$ was analyzed by stress-driven shear experiments. All rotational flow curves were obtained using steady state to prevent delay in heating, in accordance with established procedures. ${ }^{38}$

\section{Results and Discussion}

3.1. DSC. The mixed polymer-vesicle samples analyzed here lie in the one-phase region of the phase map ${ }^{21}$ where a viscoelastic weak gel-like behavior has been found (Figure 1).

The neat catanionic SDS/DDAB vesicles, at a composition of $X_{\mathrm{SDS}}=0.71$ and in the range $5-55 \mathrm{mM}$, show a chain melting transition (also known as gel-to-liquid crystal transition), as previously reported. ${ }^{39} \mathrm{An}$ interesting feature is that the main transition temperature, $T_{\mathrm{m}}$, is concentration dependent (Table 1 , values from this work), while the transition enthalpy, calculated per DDAB mole, is of about $16 \pm 1 \mathrm{~kJ} \mathrm{~mol}^{-1}$ and constant. The DSC shows a main peak with a lower temperature shoulder (or a weaker peak, for samples with low surfactant concentrations), indicating the presence of a pre-main transition. Neat DDAB vesicles ${ }^{39}$ have a melting transition at $15.7^{\circ} \mathrm{C}$, and the enthalpy involved is of the order of $25 \mathrm{~kJ} \mathrm{~mol}^{-1}$. JR400 and LM200 aqueous solutions, alone, do not show any phase transition in the studied temperature range $\left(5-50{ }^{\circ} \mathrm{C}\right)$.

In the presence of each polycation, a distinct thermal behavior is found for the catanionic vesicles, as can be seen in the thermograms in Figure 2 a,b and data in Table 1. The thermograms were run for samples at constant polymer concentration and varying surfactant concentration, and vice-versa. For the LM200-vesicle system, $T_{\mathrm{m}}$ raises as compared to the neat vesicle value, with the temperature change, $\Delta T_{\mathrm{m}}$, defined as $T_{\mathrm{m}}$ (polycation-vesicle system) $-T_{\mathrm{m}}$ (neat vesicles), ranging between +2.6 and $+3.7^{\circ} \mathrm{C}$. The opposite trend is seen for the JR400-vesicle system, where a lowering of $T_{\mathrm{m}}$ is observed, with $\Delta T_{\mathrm{m}}$ varying within -1.2 to $-4.3{ }^{\circ} \mathrm{C}$.

Another observation is that the peaks become slightly broader in the presence of the polycations. For LM200, the $\Delta T_{\mathrm{m}}$ does not vary in a clear trend with polymer or surfactant concentration. For JR400, a closer inspection of Table 1 shows that, at constant polymer concentration, $\Delta T_{\mathrm{m}}$ decreases steadily as the surfactant concentration increases. This merely reflects the fact that, in the presence of this polymer, $T_{\mathrm{m}}$ decreases only slightly with surfactant concentration, whereas for the neat vesicles the decrease is faster (first column, Table 1).

For the sample L3S8, which lies close to the precipitation phase boundary, a second peak at higher temperatures is observed (Figure 2a). This peak is related to the solubilization of amorphous polymer-surfactant crystallites (checked by light microscopy), which are kinetically trapped on the polymer network.

The enthalpy values, $\Delta_{\mathrm{m}} H$, also calculated per mole of $\mathrm{DDAB}$, have a relative large uncertainty $( \pm 10-20 \%)$ due to the broadness of peaks and ill baseline definition. We note only that $\Delta_{\mathrm{m}} H$ increase as compared with the free catanionic system, lying in the range $25-40 \mathrm{~kJ} \mathrm{~mol}^{-1}$ for the LM200-vesicle system and $20-30 \mathrm{~kJ} \mathrm{~mol}^{-1}$ for the JR400-vesicle one.

Since LM200 is both hydrophobically modified and charged, the roles of charge and of the hydrophobic graft, independently of each other, have also been probed. Table 1 and Figure $2 \mathrm{c}$ present DSC data obtained for the vesicles with added DTAB, a single-chained cationic amphiphile, and HMHEC, a neutral hydrophobically modified polymer. In both cases, the vesicles remained stable in solution when the co-solute was added, without signs of phase separation. DTAB was added to the vesicles in the same ratio as the LM200 graft/vesicle ratio and within the concentration range of polymer studied. For all DTAB - vesicle samples only one peak is observed and $\Delta T_{\mathrm{m}}$ is of the order of $+6-7{ }^{\circ} \mathrm{C}$. The situation is different with HMHEC, used as a nonionic model of LM200. The very high viscosity of the samples does not allow the exact match between the graft number in the LM200 and the HMHEC samples. Under this constraint, it is still possible to observe that the $T_{\mathrm{m}}$ of HMHEC-vesicles decreases very slightly (of about $2{ }^{\circ} \mathrm{C}$ ) with respect to the neat vesicles. Considering the enthalpy values, they are $20 \mathrm{~kJ} \mathrm{~mol}^{-1}$ for the HMHEC system, while for the 
a)

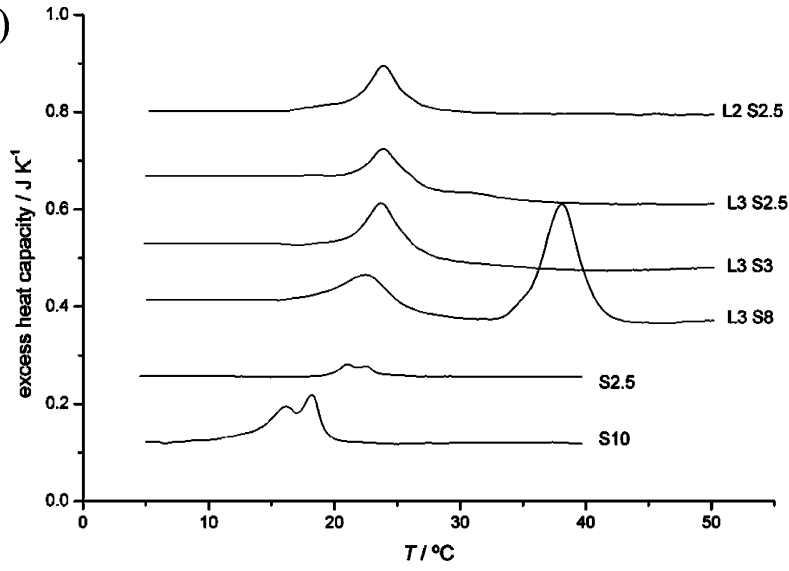

b)

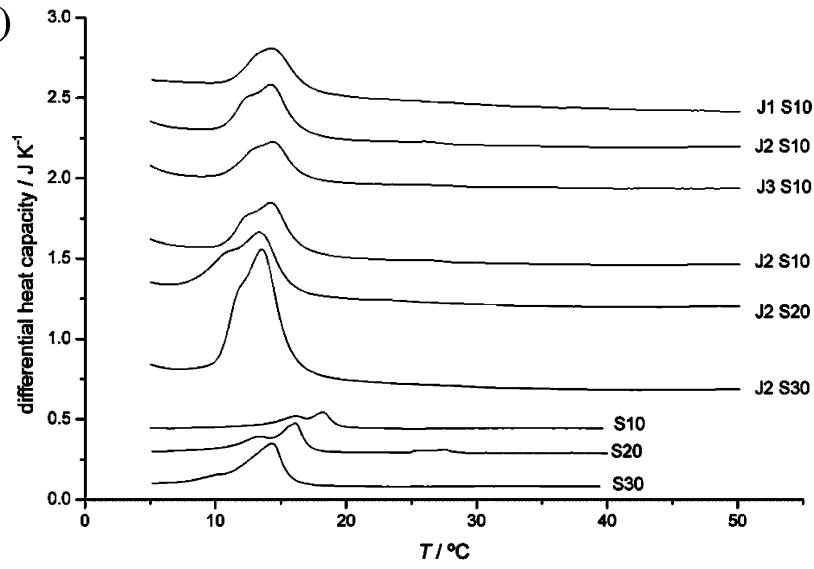

c)

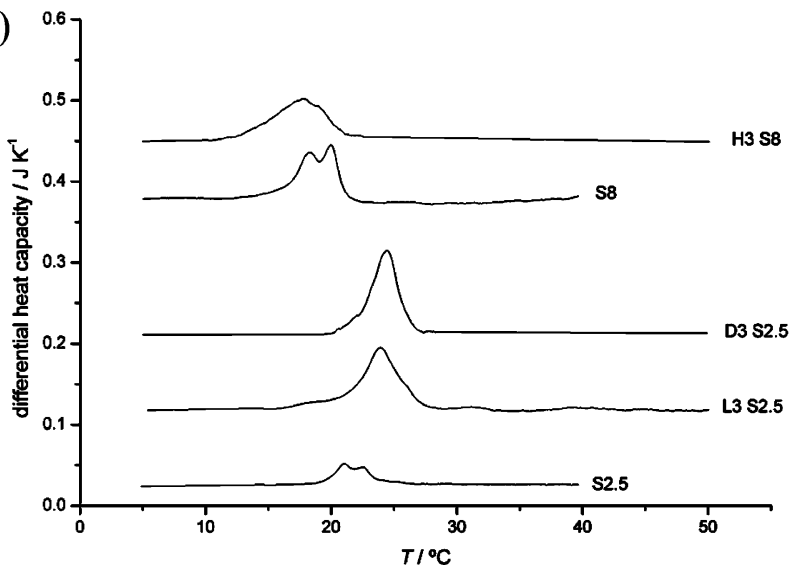

Figure 2. DSC upscans of polymer-vesicle samples investigated in this work: (a) LM200-vesicle system; (b) JR400-vesicle system; (c) vesicles in the presence of DTAB (D) and polymer HMHEC $(\mathrm{H})$. Vesicles are composed of a SDS/DDAB mixture and $X_{\mathrm{SDS}}=0.71$. Samples are labeled as Sx, LySx, or JySx with S, L, and J, where S = surfactant, $x=$ surfactant concentration in $\mathrm{mM}, \mathrm{L}=\mathrm{LM} 200, \mathrm{~J}=\mathrm{JR} 400$, and $y=$ polymer wt $\%$. Samples with HMHEC and DTAB are denoted as HySx and DySx, respectively, following the same nomenclature. Heating rate: $1.0 \mathrm{~K} \mathrm{~min}^{-1}$.

$\mathrm{DTAB}-$ vesicles they are significantly higher, in the range of $60-80 \mathrm{~kJ} \mathrm{~mol}^{-1}$.

In summary, the $\Delta T_{\mathrm{m}}$ values and $\Delta_{\mathrm{m}} H$ for LM200 indicate that the polymer induces a relative stabilization of the gel state of the vesicles (i.e., the surfactant chains crystallize upon polymer adsorption). For JR400, the homopolymer with higher charge density, the gel state is destabilized (lowering of $T_{\mathrm{m}}$ ). Furthermore, the introduction of an oppositely charged amphiphile clearly stabilizes the gel state of the vesicles (DTAB

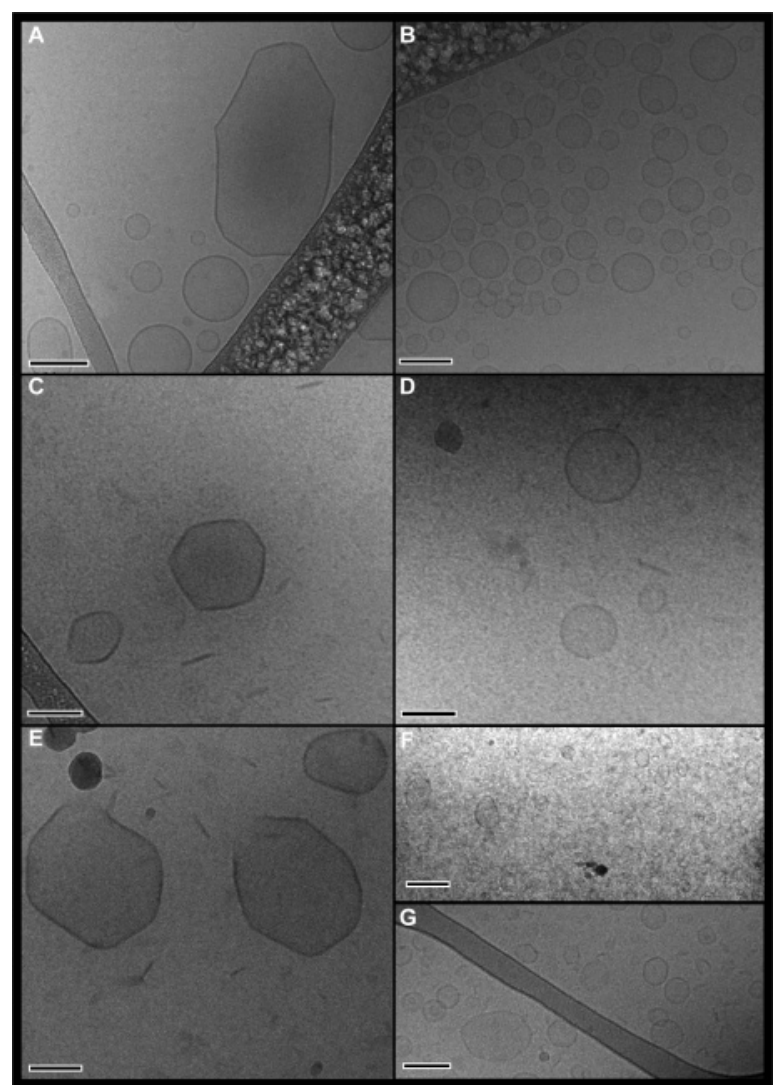

Figure 3. Cryo-TEM imaging of vesicles at temperatures below and above their chain melting temperature: (A, B) neat vesicles, sample S10, at 9 and $30{ }^{\circ} \mathrm{C}$, respectively; (C, D) LM200-vesicle system, sample L3S8, at 9 and $30{ }^{\circ} \mathrm{C}$, respectively; (E, F) JR400-vesicle system, sample J1S10, at 9 and $30^{\circ} \mathrm{C}$, respectively; (G) JR400-vesicle system, sample $\mathrm{J} 2 \mathrm{~S} 10$, at $25^{\circ} \mathrm{C}$. Samples are labeled as Sx, LySx, or JySx, where $\mathrm{S}=$ surfactant, $x=$ surfactant concentration in $\mathrm{mM}, \mathrm{L}=$ $\mathrm{LM} 200, \mathrm{~J}=\mathrm{JR} 400$, and $y=$ polymer wt $\%$.

results), whereas the hydrophobic modification of a polymer per se does not seem to favor the crystallization of the vesicles and may even marginally destabilize the gel state.

3.2. Cryo-TEM Imaging. To elucidate structural effects, direct imaging of vitrified samples of vesicles, with and without each polymer, was carried out at different temperatures. Figure $3 \mathrm{~A}, \mathrm{~B}$ shows the neat polymer-free vesicles at 9 and $30{ }^{\circ} \mathrm{C}$, respectively. Above the transition temperature, all vesicles irrespective of size have a spheroidal shape (Figure 3B). At $9^{\circ} \mathrm{C}$, below $T_{\mathrm{m}}$, the larger vesicles (range $50-100 \mathrm{~nm}$ ) appear with a faceted shape (Figure 3A), an effect directly resulting from the chain crystallization. The smaller vesicles nevertheless appear to remain spheroidal, and this effect is expected. Previous work on dioctadecyldimethylammonium bromide vesicles, for instance, has shown that vesicles with a high curvature do not crystallize even if they are cooled below $T_{\mathrm{m}} \cdot{ }^{25,40}$

LM200-vesicles observed at $9{ }^{\circ} \mathrm{C}$ are deformed and faceted in shape, and some sparse disk-like fragments also occur (Figure $3 \mathrm{C})$. At $30^{\circ} \mathrm{C}$, above their $T_{\mathrm{m}}\left(23-24{ }^{\circ} \mathrm{C}\right)$, the vesicles appear all spheroidal in shape, clearly showing that they are now in a fluid state (Figure 3D). It should be noted that imaging of this system previously done ${ }^{12,21}$ showed the presence also of faceted vesicles, together with spherical, non-faceted ones. Since these samples were vitrified at about $25^{\circ} \mathrm{C}$, i.e., very close to the chain melting temperature, both fluid and gel-phase vesicles coexisted. ${ }^{12,21}$

JR400-vesicles, below $T_{\mathrm{m}}$, also appear as faceted in shape and some disrupted, with planar fragments also occurring. 
(a)

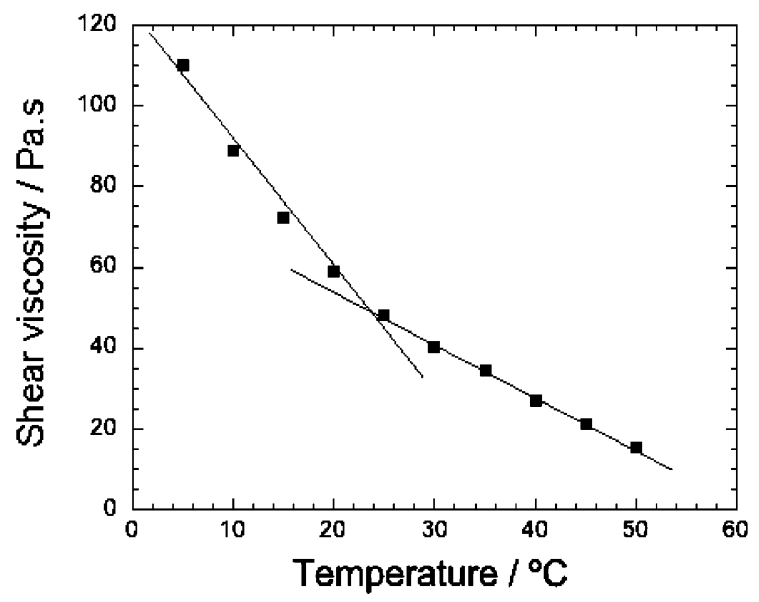

(b)

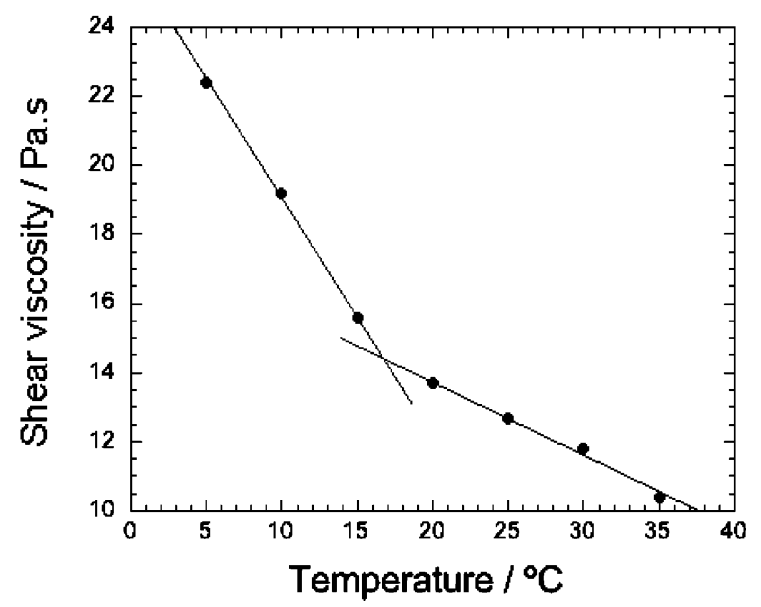

Figure 4. Dependence of shear viscosity on temperature for the polymer-vesicle samples: (a) L4S8 (4 wt \% LM200 and 8 mM of surfactant); (b) J2S10 (2 wt \% JR400 and $10 \mathrm{mM}$ surfactant). The shear rate was kept constant at $1.0 \mathrm{~s}^{-1}$. The lines are guide for the eyes.

However, a striking difference from the previous system is that at 25 and $30^{\circ} \mathrm{C}$, i.e., much above the chain melting temperature detected by DSC $\left(13-14{ }^{\circ} \mathrm{C}\right)$, the faceted shape is still seen for the vast majority of vesicles (Figure 3F,G). Furthermore, as exemplified by Figure 3F,G, individual faceted vesicles are observed throughout the different samples. This indicates that the origin of the faceting is not connected to crowding effects originated by the polymer bridging. In fact, polygonal faceting has been seen to occur for some catanionic vesicles in the presence of salt, ${ }^{41}$ as a result of close vesicle-vesicle interactions, but this is not the case here. We note further that no small micellar aggregates of any kind were observed at low or high temperature.

3.3. Rheology. Figure $4 a, b$ shows the typical temperature dependence of the shear viscosity for LM200-vesicle and JR400-vesicle samples. If one considers neat LM200 and JR400 solutions (i.e., in the absence of vesicles), the shear viscosity is orders of magnitude lower and decreases continuously with temperature without any inflection (data not shown). If vesicles are present in the polymer solution, the shear viscosity has not only much higher values but also a different temperature dependence.

It has been shown in previous work that the viscosity of lamellar or vesicular dispersions reaches a maximum at the gellc phase transition. ${ }^{27,42}$ In the polymer-vesicle samples here investigated no such maximum has been detected, due to the intrinsic viscosity of the polymer solution. Instead, a continuous and steep decrease in viscosity with temperature for both polymers is observed.

In Figure 4a, for the LM200-vesicle system, the viscosity can be seen to decrease steadily with temperature, with an inflection point at $24{ }^{\circ} \mathrm{C}$, after which there is a weaker dependence. This inflection coincides with the DSC-detected gel-to-liquid crystal transition. For the JR400 system, the viscosity varies in a similar fashion with $T$ and shows an inflection point at around $15^{\circ} \mathrm{C}$, also practically coincident with the measured $T_{\mathrm{m}}$ of the vesicles.

The strong increase in viscosity below $T_{\mathrm{m}}$ is connected with the reduction in surfactant flexibility at the molecular level when chains are in a quasi-crystalline state. The reduction in flexibility of the vesicles as a whole can be an additional or concomitant explanation, since it would induce shape deformation and thus higher viscosity. However, since the vesicles are not loose but interconnected in a network, this effect has a low impact in the rheological response. For LM200, due to the anchoring of the grafts in the vesicle bilayer, higher viscosity values are observed as compared to JR400, where only electrostatic interactions play a role. Upon cooling below $T_{\mathrm{m}}$, the viscosity increases within a broad region, instead of a deep increase. This cannot be explained by kinetic effects since the measurements were performed at steady state but would stem, instead, from the relatively high vesicle polydispersity, with larger vesicles melting at higher temperatures than the smaller ones. This interpretation is supported by the broad DSC peaks in the mixed systems.

In systems with an associating polymer and surfactant aggregates, the viscosity can be controlled by the number of active links (polymers which are bridging the aggregates) and the strength of the polymer anchoring to the surfactant aggregate. These two contributions can be followed by the storage modulus $\left(G^{\prime}\right)$ and the relaxation time $(\tau)$, respectively. For the LM200 system, the two parameters clearly show a different behavior as a function of temperature (Figures $5 \mathrm{a}$ and b), contributing in different ways to the viscosity.

By an increase of the temperature, the storage modulus $\left(G^{\prime}\right)$ decreases without any inflection around $T_{\mathrm{m}}$ (Figure 5a). This effect results from the fact that the number of cross-links is not influenced by the state of the membrane but by the concentration of vesicles. The relaxation time, instead, decreases steeply with temperature until $T_{\mathrm{m}}$ is reached, while above this value the decrease is much weaker. That is, below $T_{\mathrm{m}}$ the system needs longer time to relax and as the temperature goes down this time increases sharply. When the surfactant chains are in a quasicrystalline state (below $T_{\mathrm{m}}$ ), due to the slow dynamics, polymer anchoring in the vesicles is more long-lived than in the fluid state, where the chains are in a state of high conformational and rotational disorder and high translational mobility. The type of relaxation time curve, below $T_{\mathrm{m}}$, is not known, but it is clearly seen that a small variation in temperature in the "crystalline" phase promotes much higher changes in the relaxation time than in the liquid crystalline phase. Overall, from these observations, and considering that the viscosity depends on the relaxation times and on the storage modulus at high frequencies, it appears that the main contribution to the viscosity profile with temperature is the relaxation time. For the JR400 system, the relaxation times occur at very low frequencies and the extrapolation of $G^{\prime}$ and $G^{\prime \prime}$ curves might have significant error. Another try to reach the relaxation time was performed by a flow curve, but the usual 

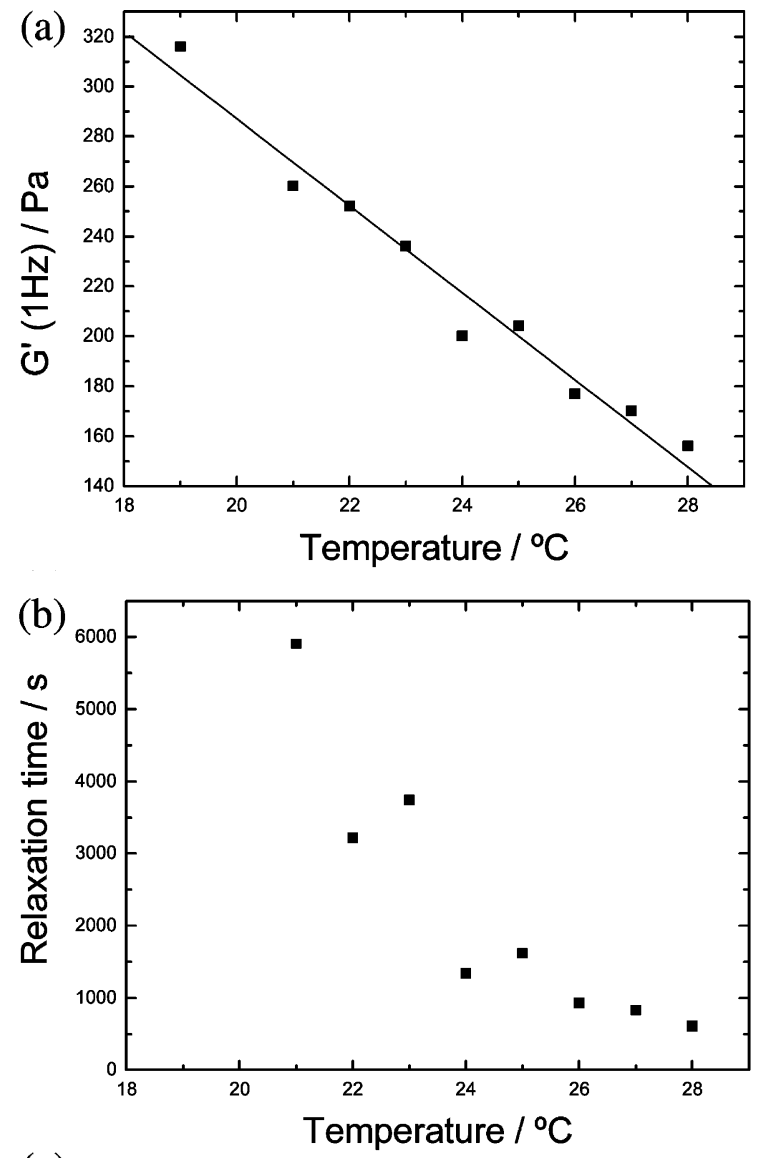

(c)

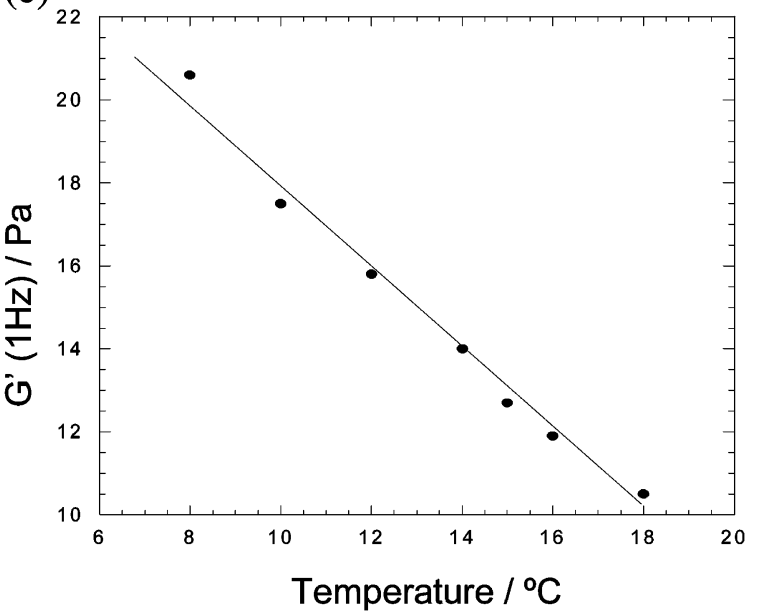

Figure 5. Temperature dependence of the storage modulus $G^{\prime}$ (a) and relaxation time $\tau$ (b), for the L3S8 sample ( $3 \mathrm{wt} \% \mathrm{HMP}^{+}$and $8 \mathrm{~mm}$ surfactant), and of $G^{\prime}$ (c), for the J1S10 sample (1 wt \% $\mathrm{P}^{+}$and 10 $\mathrm{mm}$ surfactant). The lines are guides for the eyes.

noisy regime at very low shear rates invalidated the calculation of this parameter. Therefore, the profile of the relaxation time as a function of temperature in this system is still unknown. The dependence of $G^{\prime}$ with temperature was studied, and the results pointed out to a virtually linear dependence within the temperature range investigated (Figure 5c).

3.4. Mechanisms for Polymer-Induced Changes in Vesicle Structure. From all the experimental data it is clear that the mechanisms for the interaction of LM200 and JR400 with the catanionic vesicles are different. These polymers will be henceforth designated as $\mathrm{HMP}^{+}$and $\mathrm{P}^{+}$, respectively, since they have characteristics of model polymers.
Free Catanionic Vesicles. The formation of faceted catanionic vesicles below the chain melting transition, in the absence of polymer, is an expected phenomenon. Bilayers are less flexible in the gel state than in the liquid crystalline state (due to the arrested dynamics of the alkyl chains), and hence, the curvature energy is much higher in the former state. One could envisage the stability of the faceted aggregates, imaged here, by considering that a 1:1 stoichiometric composition between cationic and anionic chains exists in the flat areas, whereas the excess anionic surfactant can accumulate in the edges where the curvature is significantly higher. In the fluid vesicles, there is complete inplane miscibility of the two components and the faceting is removed. This type of reversible segregation, concomitant with chain crystallization, has been shown to occur in salt-free catanionic vesicles, by Dubois et al. ${ }^{29}$

$\mathrm{HMP}^{+}-$Catanionic Vesicle System. The observed faceting of the $\mathrm{HMP}^{+}-$vesicles at room temperature is clearly associated to a polymer-induced crystallization of the surfactant alkyl chains. Again, once the temperature increases, the chains melt and the faceting is removed. Assuming that the segregationcrystallization mechanism is valid for the neat vesicles, the addition of $\mathrm{HMP}^{+}$is concomitant with a higher degree of formation of 1:1 stoichiometric regions, due the attraction between the excess anionic amphiphile in the free vesicles and the cationic dodecyl grafts of the polymer. This signifies that the crystalline state of the vesicles can be stabilized with respect to the fluid state; hence, $T_{\mathrm{m}}$ increases. To be noted is that in all samples in the gel-like region of the phase map (Figure 1b) there is excess polymer charge. Thus, if there is insufficient anionic surfactant to stabilize the edges of the faceted vesicles, this may give rise to vesicle breakage and formation of disklike aggregates. ${ }^{29}$ This phenomenon appears to be seen in this system as well (Figure 3C,D).

The DSC results strongly suggest, further, that the mechanism behind the $T_{\mathrm{m}}$ increase is not related primarily with the simple anchorage of the polymer hydrophobic grafts in the bilayer, since the polymer with uncharged grafts (HMHEC) does not induce crystalization effects. The grafts per se may induce vesicle bridging and a dramatic viscosity increase in the system, but it is the associated charge which is responsible for the crystallization effect and hence the reshaping. Controlled variation of the degree of modification (and thus charge density) of the $\mathrm{HMP}^{+}$system should further elucidate the proposed mechanism.

$P^{+}-$Catanionic Vesicle System. With respect to the nonmodified polymer case, the picture seems to be somewhat more complex. $\mathrm{P}^{+}$does not bear any hydrophobic modification and possesses a higher charge density than $\mathrm{HMP}^{+}$. The chain melting temperature decreases with respect to the neat vesicles (and is thus significantly lower than the mixed $\mathrm{HMP}^{+}$-vesicles). Furthermore, the faceted deformation occurs both for vesicles below and above their $T_{\mathrm{m}}$. In our view, the most likely explanation for these observations is to consider a polymerinduced charge polarization of the membrane and, thus, in-plane segregation of the two vesicle components.

It is reasonable to assume that the addition of a polycation of high charge density can induce changes on a cationic-anionic vesicle on the basis of charge polarization. In our system this would translate into the formation of the following: anionic surfactant-rich domains in the bilayer, where the polymer is strongly associated, due to strong electrostatic attractions and favorable polymer counterion entropy, and where the bilayer curvature is low; polymer-depleted cationic surfactant-rich domains. Such mechanism is consistent with several observations in our work. First, it explains the observed decrease in 
the $T_{\mathrm{m}}$ of the $\mathrm{P}^{+}-$vesicles, as the latter would be associated with the chain crystallization of the cationic rich domains. Second, it is consistent with the dominance of faceted vesicles observed even above the $T_{\mathrm{m}}$, since polarization could still induce the low-curvature facets (polycation-anionic patches). The fluid cationic rich patches could now more easily adjust to the higher curvature in the contact edges and stabilize the deformed shape. Third, the extremely high cross-link lifetimes found at $25{ }^{\circ} \mathrm{C}$ (above $T_{\mathrm{m}}$ ), reported in previous work, ${ }^{12}$ could also be explained by the existence of regions of strong polycation-surfactant association. Further support for this mechanism comes from Monte Carlo simulations carried out by Dias et al., which have shown that adsorption of a flexible polyion can promote spatial polarization of weakly charged or neutral catanionic membranes, for the case of freely mobile charges. ${ }^{19}$

For the samples examined here, a "complete" polarizationsegregation of the two surfactants leading to vesicle disruption does not seem to occur in a significant extent. That would imply reformation of polymer/anionic rich aggregates and of polymerfree DDAB-rich vesicles, which would be spheroidal above their melting temperature ${ }^{21}$ and typically larger ${ }^{21}$ than those observed here. However, we note that, for samples lying closer to the precipitation region observed in previous work, ${ }^{21}$ disk-like aggregates coexist with both faceted and spheroidal vesicles above $T_{\mathrm{m}}$, implying that a vesicle breaking mechanism could be more significant when charge neutralization is approached.

We also point out again that polygonally shaped faceted vesicles (presumably above their $T_{\mathrm{m}}$ ), with two juxtaposed bilayers, have been observed in a catanionic system, in the presence of high amount of salt. ${ }^{41}$ In this case, the salt induces strong attractive interactions and the deformation stems directly from the vesicle clustering, i.e., close vesicle-vesicle interactions. ${ }^{41}$ This type of mechanism where the role of salt could be replaced with the polycation does not take place here, since individual faceted vesicles are observed in various samples at different temperatures above $T_{\mathrm{m}}$.

One can speculate that the type of shape effects and interaction mechanism discussed here is highly dependent on $\mathrm{P}^{+}$charge density. For high enough charge density, complete segregation would be favored and the vesicles would break, presumably into polymer/anionic surfactant (disk-like) aggregates and spheroidal fluid vesicles enriched in the cationic surfactant. If too low, polarization-segregation effects in the fluid state, where the surfactants chains have large lateral mobility, would not be favored and faceting would not occur above $T_{\mathrm{m}}$. To bring further insight to this problem, the variation of the charge density of the unmodified polycation in the mixed polymer-vesicle systems is currently under investigation.

\section{Concluding Remarks}

The mechanisms behind the faceting of vesicles in polymercatanionic vesicles have been investigated, where the polymer is oppositely charged to the vesicle and bears or not a grafted chain. DSC, cryo-TEM, and viscosity results altogether show that the faceting involves different mechanisms, depending on polymer architecture.

For $\mathrm{HMP}^{+}$, with a lower charge density, the observed effect in the mixed samples is that of vesicle faceting and a stabilization of the vesicle gel phase, with an increase of the chain melting temperature. The insertion of a hydrophobic graft in the vesicle increases the degree of connectivity of the system (through vesicle bridging) and thus the viscosity but does not promote by itself crystallization, since the graft has to be charged. A crystallization-segregation mechanism of surfactant alkyl chains induced by the polycation is proposed.
For $\mathrm{P}^{+}$, more densely charged and without grafts, the faceting remains even after the vesicle phase transition, and the main consequence is a reduction in viscosity for the system. Here, a charge polarization-lateral segregation mechanism is proposed.

Acknowledgment. F.E.A. and R.O.B. acknowledge the Fundação para Ciência e Tecnologia (FCT) for Ph.D. research grants, Ref SFRH/BD/8168/2002 and Ref SFRH/BD/16380/ 2004, respectively. E.F.M. also acknowledges financial support from FCT through CIQ(UP)-Linha 5 and FCT/FEDER through the research project POCTI/QUI/ 44296/2002. F.E.A., B.L., and M.M. also acknowledge the FCT/FEDER through Projects POCTI/QUI/35415 and POCI/QUI/58689/2004.

\section{References and Notes}

(1) Stauch, O.; Ulhman, T.; Frolich, M.; Thomann, R.; El-Badry, M.; Kim, Y. -K.; Schubert, R. Biomacromolecules 2002, 3, 324-332.

(2) Baenziger, J. E.; Morris, M. L.; Darsaut, T. E.; Ryan, S. E. J. Biol. Chem. 2000, 275, 777-784.

(3) Cantor, R. S. Biochemistry 1997, 36, 2339-2344

(4) da Costa, C. J. B.; Ogrel, A. A.; McCardy, E. A.; Blanton, M. P.; Baenziger, J. E. J. Biol. Chem. 2002, 277, 201-208.

(5) Dan, N.; Safran, S. A. Biophys. J. 1998, 75, 1410-1414.

(6) Poveda, J. A.; Encinar, J. A.; Fernandez, A. M.; Mateo, C. R. Ferragut, J. A.; Gonzalez-Ros, J. M. Biochemistry 2002, 41, 12253-12262.

(7) Lasic, D. D. Trends Biotechnol. 1998, 16, 307-321.

(8) Kono, K. Adv. Drug Delivery Rev. 2001, 53, 307-319.

(9) Lasic, D. Liposomes in gene delivery; CRC Press: Boca Raton, FL, 1997.

(10) Barenholz, Y. Curr. Opin. Colloid Interface Sci. 2001, 6, 66-77. (11) Loyen, K.; Iliopoulos, I.; Audebert, R.; Olsson, U. Langmuir 1995, $11,1053-1056$

(12) Antunes, F. E.; Marques, E. F.; Gomes, R.; Thuresson, K.; Lindman, B.; Miguel, M. G. Langmuir 2004, 20, 4647-4656.

(13) Bordi, F.; Cametti, C.; Diociaiuti, M.; Gaudino, D.; Gili, T.; Sennato, S. Langmuir 2004, 20, 5214-5222.

(14) Hartmann, W.; Galla, H.-J. Biochim. Biophys. Acta 1978, 509, 474482 .

(15) Laroche, G.; Pezolet, M.; Dufourcq, J.; Dufourcq, E. Prog. Colloid Polym. Sci. 1989, 79, 38-43.

(16) Harden, J. L.; MacKintosh, F. C.; Olmsted, P. D. Phys. Rev. E 2005, 72, 11903-1-11903-13.

(17) Diederich, A.; Bähr, G.; Winterhalter, M. Langmuir 1998, 4597.

(18) Crowell, K. V.; Macdonald, P. M. J. Phys. Chem. B 1998, 102 9091-9100.

(19) Dias, R. S.; Pais, A. A. C. C.; Linse, P.; Miguel, M. G.; Lindman, B. J. Phys. Chem. B 2005, 109, 11781-11788.

(20) Meier, W. Langmuir 1996, 12, 6341-6345.

(21) Marques, E. F.; Regev, O.; Khan, A.; Miguel, M. D.; Lindman, B. Macromolecules 1999, 32, 6626-6637.

(22) Lee, J. H.; Gustin, J. P.; Chen, T. H.; Payne, G. F.; Raghavan, S. R. Langmuir 2005, 21, 26-33.

(23) Medronho, B.; Antunes, F. E.; Lindman, B.; Miguel, M. G. J Dispersion Sci. Technol. 2006, 27, 83-90.

(24) Andersson, M.; Hammarstrom, L.; Edwards, K. J. Phys. Chem. 1995, 99, 14531-14538

(25) Cocquyt, J.; Olsson, U.; Olofsson, G.; Van, der Meeren, P. Langmuir 2004, 20, 3906-3912.

(26) Oliger, P.; Schmutz, M.; Hebrant, M.; Grison, C.; Coutrot, P.; Tondre, C. Langmuir 2001, 17, 3893-3897.

(27) Vautrin, C.; Zemb, T.; Schneider, M.; Tanaka, M. J. Phys. Chem. B 2004, 108, 7986-7991.

(28) Dubois, M.; Deme, B.; Gulik-Krzywicki, T.; Dedieu, J.-C.; Vautrin, C.; Desert, S.; Perez, E.; Zemb, T. Nature 2001, 411, 672-675.

(29) Dubois, M.; Lizunov, V.; Meister, A.; Gulik-Krzywicki, T.; Verbavatz, J. M.; Perez, E.; Zimmerberg, J.; Zemb, T. Proc. Natl. Acad. Sci. (U.S.A.) 2004, 101, 15082-15087.

(30) Marques, E. F.; Regev, O.; Khan, A.; Miguel, M. D.; Lindman, B. J. Phys. Chem. B 1998, 102, 6746-6758.

(31) Kaler, E. W.; Herrington, K. L.; Murthy, A. K.; Zasadzinski, J. A. J. Phys. Chem. 1992, 96, 6698-6707.

(32) Tondre, C.; Caillet, C. Adv. Colloid Interface Sci. 2001, 93, 115134.

(33) Thuresson, K.; Nilsson, S., Lindman, B. Langmuir 1996, 12, 530537.

(34) Dhoot, S.; Goddard, E. D.; Murphy, D. S.; Tirrell, M. Colloids Surf. 1992, 66, 91-96. 
(35) Guillemet, F.; Piculell, L. J. Phys. Chem. 1995, 99, 92019209.

(36) Bellare, J. R.; Davis, H. T.; Scriven, L. E.; Talmon, Y. J. Electron Microsc. Tech. 1988, 10, 87-111.

(37) Barnes, H. A. H., J. F.; Walters, K. An Introduction to Rheology; Elsevier: Amsterdam, 1989.

(38) Karlson, L.; Nilsson, S.; Thuresson, K. Colloid Polym. Sci. 1999, 277, 798-804.
(39) Marques, E. F.; Khan, A.; Lindman, B. Thermochim. Acta 2002 394, 31-37.

(40) Brito, R. O.; Marques, E. F. Chem. Phys. Lipids 2005, 137, $18-$ 28.

(41) Jung, H. T.; Coldren, B.; Zasadzinski, J. A.; Iampietro, D. J.; Kaler, E. W. Proc. Natl. Acad. Sci. (U.S.A.) 2001, 98, 1353-1357.

(42) Benatti, C. R.; Tiera, M. J.; Feitosa, E.; Olofsson, G. Thermochim Acta 1999, 328, 137-142. 